\title{
Article
}

\section{"My Life during the Lockdown": Emotional Experiences of European Adolescents during the COVID-19 Crisis}

\author{
Alberto Forte ${ }^{1,2, *}$, Massimiliano Orri ${ }^{3,4}$, Martina Brandizzi ${ }^{5}$, Cecilia Iannaco ${ }^{1}$, Paola Venturini ${ }^{1,5}$, \\ Daniela Liberato ${ }^{1}$, Claudia Battaglia ${ }^{1,6}$, Isabel Nöthen-Garunja ${ }^{1}$, Maria Vulcan ${ }^{7}$, Asja Brusìc ${ }^{8}$, \\ Lauro Quadrana ${ }^{6,9}$, Olivia Cox ${ }^{10}$, Sara Fabbri ${ }^{10}$ and Elena Monducci ${ }^{11}$
}

Citation: Forte, A.; Orri, M.; Brandizzi, M.; Iannaco, C.; Venturini, P.; Liberato, D.; Battaglia, C.; Nöthen-Garunja, I.; Vulcan, M.; Brusìc, A.; et al. "My Life during the Lockdown": Emotional Experiences of European Adolescents during the COVID-19 Crisis. Int. J. Environ. Res. Public Health 2021, 18, 7638. https:/ / doi.org/10.3390/ijerph18147638

Academic Editors: Davide Gori and Federica Guaraldi

Received: 19 June 2021

Accepted: 13 July 2021

Published: 18 July 2021

Publisher's Note: MDPI stays neutral with regard to jurisdictional claims in published maps and institutional affiliations.

Copyright: (c) 2021 by the authors Licensee MDPI, Basel, Switzerland. This article is an open access article distributed under the terms and conditions of the Creative Commons Attribution (CC BY) license (https:/ / creativecommons.org/licenses/by/ $4.0 /)$
1 European Network for Psychodynamic Psychiatry, 00154 Rome, Italy; cecilia.iannaco@netforpp.eu (C.I.); paola.venturini@aslroma1.it (P.V.); daniela.liberato@netforpp.eu (D.L.); claudia.battaglia@uniroma1.it (C.B.); isabel.noethengarunja@netforpp.eu (I.N.-G.)

2 Department of Psychiatry and Substance Abuse, ASL Roma 5, 00019 Rome, Italy

3 McGill Group for Suicide Studies, Douglas Mental Health University Institute, Department of Psychiatry, McGill University, Montreal, QC H3A 0G4, Canada; massimiliano.orri@mail.mcgill.ca

4 Bordeaux Population Health Research Centre, Inserm U1219, Université de Bordeaux, 30072 Bordeaux, France

5 Department of Psychiatry, ASL Roma 1, 00193 Rome, Italy; martina.brandizzi@aslroma1.it

6 Policlinico Umberto I, 00185 Rome, Italy; lauro.quadrana@uniroma1.it

7 Timișoara 2021-European Capital of Culture Association, 300057 Timișoara, Romania; maria.vulcan@timisoara2021.ro

8 Rijeka 2020_European Capital of Culture, Croatian Cultural Centre, 51000 Rijeka, Croatia; Asja@hkd-rijeka.hr

9 Department of Neurosciences and Mental Health, Section of Child and Adolescent Neuropsychiatry, Sapienza University of Rome, 00185 Rome, Italy

10 Faculty of Medicine and Psychology, Sapienza University, 00185 Rome, Italy; ollicox93@gmail.com (O.C.); sarafabbri92@gmail.com (S.F.)

11 Department of Human Neurosciences, Sapienza University, 00185 Rome, Italy; elena.monducci@uniroma1.it

* Correspondence: alberto.forte@uniroma1.it

Abstract: This study investigates, using an online self-report questionnaire, adolescents' emotional reactions during the lockdown in a sample of 2105 secondary school students (aged 14-19) in Italy, Romania, and Croatia. We used a self-reported online questionnaire (answers on a 5-point scale or binary), composed of 73 questions investigating the opinions, feelings, and emotions of teenagers, along with sociodemographic information and measures of the exposure to lockdown. The survey was conducted online through a web platform in Italy (between 27 April and 15 June 2020), Romania, and Croatia ( 3 June and 2 July 2020). Students aged $>14$ years, living in a small flat, and not spending time outside were more likely to report anger, sadness, boredom/emptiness, and anxiety. Boys were significantly less likely than girls to report all measured emotional reactions. Those who lost someone from COVID-19 were more than twice as likely to experience anger compared to those who did not. Our findings may help identifying adolescents more likely to report negative emotional reactions during the COVID-19 pandemic and inform public health strategies for improving mental health among adolescents during/after the COVID-19 crisis.

Keywords: adolescents; lockdown; emotional reactions; school-students

\section{Introduction}

The COVID-19 epidemic started in China in December 2019 and rapidly spread worldwide [1]. To respond to the public health crisis, many countries introduced severe lockdown measures such as school closure, social distancing, interruption of sports activities, and quarantine/isolation [1]. These restrictive measures may have serious psychological consequences in young individuals [2], especially among the more vulnerable.

Moreover, according to UNESCO, schools were closed in 188 countries between April and May 2019, leaving about 1.5 billion students out of the school system, representing 
$60 \%$ of the world student population [3]. School closures and decreased educational opportunities due to the pandemic might have a great impact on youths' mental health [4], exposing young individuals to a higher risk of physical and/or sexual violence at home and, together with the economic damage caused by the crisis, may lead to increased mental health issues and suicide rates [5]. Furthermore, a recent systematic review of the literature showed a strong association between isolation/loneliness and depression in children and adolescents [6]. Additionally, schools play a fundamental role in the prevention and early recognition of mental disorders [7], especially for those with social and economic disadvantages [8], adding to the potential negative impact of COVID-19 on youth mental health.

Recent studies have shown increased levels of depressive, anxiety, and post-traumatic stress symptoms among adolescents in the context of the COVID-19 pandemic [9], particularly among girls, senior high school/college students, and those with economic disadvantages [6,10-14]. However, only a few of them focused on the emotional reactions of adolescents during the COVID-19 outbreak [15] and on psychological distress [16-18]. Understanding the emotional reactions of secondary school students during the lockdown might help us to identify students at risk of psychological distress and inform preventive actions. In this study, we developed an online survey involving secondary school students from three European countries (Italy, Croatia, and Romania). The aim was to investigate the associations between several sociodemographic and lockdown-related factors with several emotional reactions (anger, anxiety, sadness, and boredom/emptiness).

\section{Materials and Methods}

\subsection{Study Design}

An international cross-sectional study was performed in three European countries (Italy, Romania, and Croatia) based on a questionnaire developed by the Department of Human Neurosciences/Section for Child and Adolescent Psychiatry, Sapienza University (Rome, Italy) and distributed by The European Network for Psychodynamic Psychiatry (Netforpp Europa, Rome, Italy) a non-profit organization, during and immediately after the lockdown.

\subsection{Recruitment Procedure}

As described elsewhere [19] the survey was conducted online through a web platform (SurveyMonkey ${ }^{\circledR}$ ) in Italy (between 27 April and 15 June 2020), Romania, and Croatia (between 3 June and 2 July 2020). Data collection was coordinated by Netforpp Europa in Italy and the associations Timisoara 2021 in Romania and Hrvatski Kulturi Dom in Croatia. The survey was conducted in a population of secondary school students as an extension of a previous project on mental health literacy, which had been conceived and implemented by Netforpp Europa, in collaboration with the Department of School Services of Rome (Italy), in several high schools in the cities of Rome and Florence in January 2020. Schools were recruited based on a previous EU funded project "Mai Più Memory against Inhumanity: People with Mental Disorders under Totalitarian Regimes in Europe", which involved several schools in different European countries [20].

The Croatian coordinator collaborated with the Departments of Education and Schooling of the City of Rijeka and Primorsko-goranska County, which distributed the questionnaire among schools in Rijeka and its region. In order to obtain a nationwide coverage and adequate distribution of age groups, the coordinator in Romania collaborated with the "Europe Direct" network of information centers in Arad and Bucharest, the Timis Sibiu County School Inspectorates and several media partners. About half $(n=508)$ of the total number of 1004 responses in Romania was collected in the "Elena Ghiba Birta" National College in Arad. The other half of responses came from schools in Timișoara, Sibiu, and Resifa.

Every school was contacted 2 weeks before the survey started and the study protocol was outlined in detail to head and class teachers. In Italy, approximately 7500 students 
were invited to participate (response rate $12.3 \%$ ). Each participant of the questionnaire remained anonymous and respondents' IP addresses could not be disclosed. Participation was entirely voluntary and without any compensation. Participants over the age of 18 years gave their voluntary informed consent to participate in the research before taking part in the study. For students under 18 years of age, participating schools entered the questionnaire in the school's electronic parental platform, together with a cover letter in which all the information on the study protocol and the survey's objectives were given. Schools included in the study uploaded the questionnaire to the school electronic parental platform along with a cover letter explaining in detail the study protocol. The letter contained the purpose of the study, the study procedure, and information about data protection and privacy. The school electronic parenting platform is an electronic classroom-board that can only be accessed by parents with a personal password. Only after parental acknowledgment and consent were their children able to complete the questionnaire.

\subsection{Measures}

"My life during lockdown" is a self-reported online questionnaire, which is composed of 73 questions investigating the opinions, feelings, and emotions of teenagers, along with sociodemographic information and measures of the exposure to lockdown. Types of questions differed within the survey, and were either on a 5-point Likert scale or binary questions. The questionnaire was completed anonymously, and the respondents IP address was hidden. The questionnaire was developed in Italian and translated into the Romanian, Croatian, and English languages using a translation and back-translation procedure. Measured variables are listed in Table 1. We classified municipalities into three degrees of urbanization, according to EUROSTAT [21]; metropolitan areas (cities/large urban areas), medium-size urban areas (towns and suburbs/small urban areas), and rural areas. Parents socioeconomic status (SES) were classified according their occupation into low (i.e., (unskilled manual, non-manual low, farmer, fisher, retired/social welfare, or nonworkers) vs. non-low (i.e., non-manual high, employee, self-employed and skilled worker, skilled manual, professional/managerial, or technical/skilled) [22]. Housing characteristics considered were: size of the house/apartment $\left(>\right.$ or $<60 \mathrm{~m}^{2}$ ), possibility of spending time outside, and whether it was possible to have privacy in the apartment during the lockdown (binary questions). We also asked about COVID-19-related information ("Has somebody important to you contracted SARS-CoV2?" "Have you experienced the loss of a loved one because of SARS-CoV2?"). Moreover, we asked whether parents were still working during the lockdown (answers: yes, no but they kept their job, no and their have lost their job), and whether they were worrying for the economic situation ("Are you worried about economic problems during this period?"). We also included questions about: relationships ("Has your relationship with your parents/friends/partner changed during the lockdown?", answered as no, yes negatively, yes positively); social media ("Has your use of social media increased during the lockdown?", answered on a 5-point scale ranging from Not at all to A lot); the importance of family or professional support ("Do you think that being with your family helped you to overcome this period?", "Have you ever thought it would help you to talk to a professional about your feelings?"); and the positive impact of the lockdown ("Did you discover new interests/hobbies or talents during this period?", "Do you think that you have spent this period of time in a productive and creative way?"). Finally, students' emotional reactions were assessed by asking students if they were experiencing feelings of anxiety, sadness, anger, and persistent boredom/emptiness during the lockdown period (binary questions).

\subsection{Data Analysis}

The study variables were described using mean and standard deviation (for continuous variables) and count and percentage (for categorical variables). First, descriptive statistics were estimated in the whole sample and by country, and the comparison across countries was performed using t-tests and chi-square tests. Second, associations of sociode- 
mographic and lockdown-related variables with the four measured emotional reactions were estimated using univariable logistic regressions. Third, the variables that were associated with the emotional reactions at $p<0.05$ were entered in multivariable logistic regression models to estimate their independent associations with emotional reactions. All tests were 2-tailed and considered statistically significant at $p<0.05$.

Table 1. Characteristics of the Sample.

\begin{tabular}{|c|c|c|c|c|c|c|}
\hline & Category & $\begin{array}{l}\text { Whole } \\
\text { Sample } \\
\text { (2105) }\end{array}$ & $\begin{array}{l}\text { Italy } \\
(928)\end{array}$ & $\underset{(\mathbf{1 0 0 4 )}}{\text { Romania }}$ & $\begin{array}{c}\text { Croatia } \\
(\mathbf{1 7 3 )}\end{array}$ & $\begin{array}{c}p \\
\text { Values }\end{array}$ \\
\hline \multicolumn{7}{|l|}{$\begin{array}{c}\text { Sociodemographic } \\
\text { variables }\end{array}$} \\
\hline \multirow[t]{3}{*}{ Age } & $<14$ & 415 (19.7) & $140(15.1)$ & $232(23.1)$ & $43(24.9)$ & $<0.001$ \\
\hline & $>16$ & 755 (35.9) & $335(36.1)$ & 367 (36.6) & $53(30.6)$ & \\
\hline & $14-16$ & $935(44.4)$ & $453(48.8)$ & $405(40.3)$ & 77 (44.5) & \\
\hline \multirow[t]{2}{*}{ Gender } & Girls & $1446(68.7)$ & $694(74.8)$ & $638(63.5)$ & $114(65.9)$ & $<0.001$ \\
\hline & Boys & $659(31.3)$ & $234(25.2)$ & $366(36.5)$ & $59(34.1)$ & \\
\hline \multirow[t]{3}{*}{ Degree of urbanization } & Densely populated area & $1837(87.3)$ & $905(97.5)$ & $848(84.5)$ & $84(48.6)$ & $<0.001$ \\
\hline & Intermediate density areas & $164(7.8)$ & $23(2.5)$ & $98(9.8)$ & $43(24.9)$ & \\
\hline & Rural & $104(4.9)$ & $0(0.0)$ & $58(5.8)$ & $46(26.6)$ & \\
\hline \multirow{3}{*}{ Siblings } & No & $590(28.0)$ & $206(22.2)$ & $342(34.1)$ & $42(24.3)$ & $<0.001$ \\
\hline & One & $1104(52.4)$ & $530(57.1)$ & 481 (47.9) & $93(53.8)$ & \\
\hline & More than one & $411(19.5)$ & $192(20.7)$ & $181(18.0)$ & $38(22.0)$ & \\
\hline \multirow[t]{2}{*}{ Mother low SES } & Yes & $1318(62.6)$ & $676(72.8)$ & $545(54.3)$ & $97(56.1)$ & $<0.001$ \\
\hline & No & $787(37.4)$ & $252(27.2)$ & $459(45.7)$ & $76(43.9)$ & \\
\hline \multirow{2}{*}{ Father low SES } & Yes & $526(25.0)$ & $95(10.2)$ & $335(33.4)$ & $96(55.5)$ & $<0.001$ \\
\hline & No & $1579(75.0)$ & $833(89.8)$ & $669(66.6)$ & $77(44.5)$ & \\
\hline \multicolumn{7}{|l|}{ Housing } \\
\hline \multirow[b]{3}{*}{ Time outside home } & $>60 \mathrm{~m}^{2}$ & $1825(86.7)$ & $848(91.4)$ & $829(82.6)$ & $148(85.5)$ & $<0.001$ \\
\hline & $<60 \mathrm{~m}^{2}$ & $280(13.3)$ & $80(8.6)$ & $175(17.4)$ & $25(14.5)$ & \\
\hline & Yes & $1590(75.5)$ & $648(69.8)$ & $795(79.2)$ & $147(85.0)$ & $<0.001$ \\
\hline & No & $515(24.5)$ & $280(30.2)$ & $209(20.8)$ & $26(15.0)$ & \\
\hline \multirow{2}{*}{ Privacy } & Yes & $1778(84.5)$ & $775(83.5)$ & $847(84.4)$ & $156(90.2)$ & 0.084 \\
\hline & No & 327 (15.5) & $153(16.5)$ & 157 (15.6) & $17(9.8)$ & \\
\hline \multirow{3}{*}{$\begin{array}{l}\text { COVID-19-related } \\
\text { Loved one with COVID }\end{array}$} & & & & & & \\
\hline & Yes & $141(6.7)$ & $80(8.6)$ & $56(5.6)$ & $5(2.9)$ & 0.003 \\
\hline & No & 1964 (93.3) & $848(91.4)$ & $948(94.4)$ & $168(97.1)$ & \\
\hline \multirow{2}{*}{$\begin{array}{l}\text { Loved one died of } \\
\text { COVID }\end{array}$} & Yes & $32(1.5)$ & $23(2.5)$ & $9(0.9)$ & $0(0.0)$ & 0.004 \\
\hline & No & $2073(98.5)$ & $905(97.5)$ & $995(99.1)$ & $173(100.0)$ & \\
\hline \multirow{2}{*}{ Fear of getting COVID } & Yes & $987(46.9)$ & $469(50.5)$ & $470(46.8)$ & $48(27.7)$ & $<0.001$ \\
\hline & No & $1118(53.1)$ & $459(49.5)$ & $534(53.2)$ & $125(72.3)$ & \\
\hline \multirow{3}{*}{$\begin{array}{l}\text { Trust in the } \\
\text { government }\end{array}$} & No & 375 (17.8) & $158(17.0)$ & $182(18.1)$ & $35(20.2)$ & $<0.001$ \\
\hline & Yes, enough & $1492(70.9)$ & $675(72.7)$ & $715(71.2)$ & $102(59.0)$ & \\
\hline & Yes, fully & 238 (11.3) & $95(10.2)$ & $107(10.7)$ & $36(20.8)$ & \\
\hline \multirow{4}{*}{$\begin{array}{l}\text { Job/economy } \\
\text { Parents currently } \\
\text { working }\end{array}$} & & & & & & \\
\hline & Yes & $1592(75.6)$ & $689(74.2)$ & $774(77.1)$ & $129(74.6)$ & 0.014 \\
\hline & No, kept job & $457(21.7)$ & $216(23.3)$ & $208(20.7)$ & $33(19.1)$ & \\
\hline & No, lost job & $56(2.7)$ & $23(2.5)$ & $22(2.2)$ & $11(6.4)$ & \\
\hline Worries about money * & & $3.58(1.08)$ & $3.31(1.07)$ & $3.78(1.03)$ & $3.91(1.07)$ & $<0.001$ \\
\hline \multirow{4}{*}{$\begin{array}{c}\text { Relationships } \\
\text { Changed relationship } \\
\text { parents }\end{array}$} & & & & & & \\
\hline & No & $509(24.2)$ & $175(18.9)$ & $291(29.0)$ & $43(24.9)$ & $<0.001$ \\
\hline & Positive & $1298(61.7)$ & $585(63.0)$ & $597(59.5)$ & $116(67.1)$ & \\
\hline & Negative & $298(14.2)$ & $168(18.1)$ & $116(11.6)$ & $14(8.1)$ & \\
\hline \multirow{3}{*}{$\begin{array}{l}\text { Changed relationship } \\
\text { friends }\end{array}$} & No & $474(22.5)$ & $179(19.3)$ & $258(25.7)$ & $37(21.4)$ & $<0.001$ \\
\hline & Positive & 1088 (51.7) & 525 (56.6) & $462(46.0)$ & $101(58.4)$ & \\
\hline & Negative & $543(25.8)$ & $224(24.1)$ & $284(28.3)$ & $35(20.2)$ & \\
\hline \multirow{3}{*}{$\begin{array}{l}\text { Changed relationship } \\
\text { partner }\end{array}$} & No/no partner & $1287(61.1)$ & $594(64.0)$ & $669(66.6)$ & $24(13.9)$ & $<0.001$ \\
\hline & Positive & $396(18.8)$ & 177 (19.1) & $205(20.4)$ & $14(8.1)$ & \\
\hline & Negative & $422(20.0)$ & $157(16.9)$ & 130 (12.9) & 135 (78.0) & \\
\hline
\end{tabular}


Table 1. Cont.

\begin{tabular}{|c|c|c|c|c|c|c|}
\hline & Category & $\begin{array}{c}\text { Whole } \\
\text { Sample } \\
\text { (2105) }\end{array}$ & $\begin{array}{l}\text { Italy } \\
\text { (928) }\end{array}$ & $\underset{(\mathbf{1 0 0 4 )}}{\operatorname{Romania}}$ & $\begin{array}{c}\text { Croatia } \\
\text { (173) }\end{array}$ & $\begin{array}{l}p \\
\text { Values }\end{array}$ \\
\hline $\begin{array}{c}\text { Social media } \\
\text { Increased social media } \\
\text { use }\end{array}$ & & $2.55(1.20)$ & $2.33(1.13)$ & $2.69(1.21)$ & $2.87(1.32)$ & $<0.001$ \\
\hline $\begin{array}{c}\text { Support } \\
\text { Helpful family support } \\
\text { Professional support }\end{array}$ & $\begin{array}{c}\text { Yes } \\
\text { No } \\
\text { Already in therapy } \\
\text { Yes } \\
\text { No }\end{array}$ & $\begin{array}{c}1568(74.5) \\
537(25.5) \\
99(4.7) \\
651(30.9) \\
1355(64.4)\end{array}$ & $\begin{array}{c}681(73.4) \\
247(26.6) \\
67(7.2) \\
316(34.1) \\
545(58.7)\end{array}$ & $\begin{array}{l}749(74.6) \\
255(25.4) \\
23(2.3) \\
307(30.6) \\
674(67.1)\end{array}$ & $\begin{array}{c}138(79.8) \\
35(20.2) \\
9(5.2) \\
28(16.2) \\
136(78.6)\end{array}$ & $\begin{array}{l}0.208 \\
<0.001\end{array}$ \\
\hline $\begin{array}{l}\text { Positive impact } \\
\text { New interests } \\
\text { Creative time }\end{array}$ & $\begin{array}{l}\text { Yes } \\
\text { No } \\
\text { Yes } \\
\text { No }\end{array}$ & $\begin{array}{c}1311(62.3) \\
794(37.7) \\
1310(62.2) \\
795(37.8)\end{array}$ & $\begin{array}{l}615(66.3) \\
313(33.7) \\
599(64.5) \\
329(35.5)\end{array}$ & $\begin{array}{l}605(60.3) \\
399(39.7) \\
605(60.3) \\
399(39.7)\end{array}$ & $\begin{array}{c}91(52.6) \\
82(47.4) \\
106(61.3) \\
67(38.7)\end{array}$ & $\begin{array}{l}0.001 \\
0.146\end{array}$ \\
\hline
\end{tabular}

All variables are described as $n(\%)$, except for the variables with an * that are described as mean (SD).

\subsection{Ethical Issues}

All subjects gave their written informed consent for inclusion before they participated in the study. Participation was voluntary and without compensation. The study was conducted in accordance with the Declaration of Helsinki. Moreover, the study followed the privacy recommendation released by the Italian Ministry of Education, University, and Research (MIUR) (https:/ / www.miur.gov.it/privacy-tra-i-banchi-di-scuola (accessed on 15 July 2020)). The project received Institutional approval by the Municipality of Rome, Department of School and Education (institutional authorization number n.987 06/05/2019), and was considered in line with GDPR 2016/679 (General Data Protection Regulation).

\section{Results}

\subsection{Participants' Characteristics}

A detailed description of the participants' characteristics is presented in Table 1. Most of the participants were $14-16$ years old $(44.4 \%)$ or $16-18$ years old $(35.9 \%)$, and $68.7 \%$ were female. Of the respondents, $87.3 \%$ were from a densely populated area, and $86.7 \%$ reported living in an apartment of $>60 \mathrm{~m}^{2}$. Regarding COVID-19-related variables, $6.7 \%$ indicated knowing people with COVID-19 (family members, relatives, and/or friends), while $1.5 \%$ reported knowing someone who had died from COVID-19. Several variables significantly differed among countries. Among sociodemographic variables, the degree of urbanization significantly differed among countries $(p<0.001)$; adolescents living in rural areas were mainly from Croatia and Romania, and none were from Italy $(26.6 \%, 5.8 \%$, and $0 \%$, respectively), while Italian respondents were mainly from a densely populated area $(97.5 \%)$. Additionally, housing variables significantly differed among countries for both the house size and the time spent outside: Italian respondents were more likely to live in larger houses $(p<0.001)$ and less likely to spend time outside $(p<0.001)$. Moreover, the fear of getting infected with COVID-19 was significantly higher among Italian adolescents compared to Romanian and Croatians $(50.5 \%, 46.8 \%$, and $27.7 \%$, respectively, $p<0.001)$.

\subsection{Univariable Analyses}

Boredom/emptiness was the most frequently reported emotional reaction $(n=1504$, $71.7 \%)$ followed by sadness ( $n=1062,50.5 \%)$, anxiety $(n=786,37.3 \%)$, and anger $(n=698$, $33.2 \%$ ). Italian adolescents were more likely to report boredom/emptiness, anxiety, and sadness $(p<0.001)$, whereas no significant difference between countries emerged for anger. Among sociodemographic variables, we found that age was significantly associated with all outcomes; emotional reactions were more frequently reported by adolescents aged more than 16 years and 14-16 years, compared with those younger than 14 years (Table 2). Boys were less likely than girls to report all measured emotional reactions (anger: OR: 
0.64; 95\% CI: 0.52-0.79; sadness OR: 0.35; 95\% CI: 0.28-0.42; boredom/emptiness: OR: 0.56; 95\% CI: 0.46-0.68; and anxiety: OR: 0.42; 95\% CI: 0.34-0.52). Similarly, adolescents living in rural areas were less likely to report all emotional reactions than those living in urban areas (anger: OR: 0.51; 95\% CI: 0.31-0.83; sadness OR: 0.53; 95\% CI: $0.34-0.83$; boredom/emptiness: OR: 0.59 ; 95\% CI: $0.37-0.94$; and anxiety: OR: 0.28 ; $95 \%$ CI: $0.15-0.51$ ).

Table 2. Univariable Analysis. Logistic regression models estimating the association between each variable in column 1 and the outcomes in the last four columns (Anger, Sadness, Boredom/emptiness, and Anxiety). All analyses are adjusted for country. Boredom/emptiness has been categorized as follows: always, often $=1$; never, rarely, sometimes $=0$. Statistically significant variables are in bold.

\begin{tabular}{|c|c|c|c|c|c|}
\hline Variable & Category & $\begin{array}{c}\text { Anger } \\
\text { OR }(95 \% \text { CI })\end{array}$ & $\begin{array}{c}\text { Sadness } \\
\text { OR }(95 \% \text { CI })\end{array}$ & $\begin{array}{l}\text { Boredom/Emptiness } \\
\text { OR (95\% CI) }\end{array}$ & $\begin{array}{c}\text { Anxiety } \\
\text { OR (95\% CI) }\end{array}$ \\
\hline \multicolumn{6}{|l|}{ Sociodemographic } \\
\hline \multirow[t]{2}{*}{ Age } & $>16$ vs. $<14$ & $2.05(1.55-2.69)$ & $2.19(1.71-2.8)$ & $2.32(1.79-3.01)$ & $2.92(2.22-3.85)$ \\
\hline & $14-16$ vs. $<14$ & $1.85(1.42-2.42)$ & $1.96(1.54-2.49)$ & $1.83(1.42-2.35)$ & $2.14(1.63-2.8)$ \\
\hline Gender & Boy & $0.64(0.52-0.79)$ & $0.35(0.28-0.42)$ & $0.56(0.46-0.68)$ & $0.42(0.34-0.52)$ \\
\hline \multirow[t]{2}{*}{ Urbanization } & $\begin{array}{l}\text { Intermediate } \\
\text { density areas }\end{array}$ & $0.8(0.55-1.14)$ & $1.04(0.75-1.45)$ & 1.09 (0.78-1.53) & $0.63(0.43-0.91)$ \\
\hline & Rural & $0.51(0.31-0.83)$ & $0.53(0.34-0.83)$ & $0.59(0.37-0.94)$ & $0.28(0.15-0.51)$ \\
\hline \multirow[t]{2}{*}{ Siblings } & Yes one & $1.06(0.86-1.32)$ & $1.04(0.85-1.28)$ & $1.11(0.9-1.36)$ & $1.02(0.83-1.26)$ \\
\hline & Yes, more than one & $1.29(0.99-1.69)$ & $1.1(0.85-1.41)$ & $1.18(0.91-1.52)$ & $0.97(0.75-1.27)$ \\
\hline Mother low SES & Yes & $0.93(0.77-1.13)$ & $0.88(0.73-1.05)$ & $0.73(0.61-0.88)$ & $0.96(0.8-1.16)$ \\
\hline Father low SES & Yes & $1.1(0.88-1.38)$ & $1.03(0.84-1.27)$ & $1.08(0.87-1.34)$ & $0.99(0.79-1.24)$ \\
\hline \multicolumn{6}{|l|}{ Housing } \\
\hline House & $<60 \mathrm{~m}^{2}$ & $1.3(1.00-1.69)$ & $1.16(0.9-1.49)$ & 1.07 (0.83-1.39) & $1.23(0.94-1.59)$ \\
\hline Time outside home & No & $1.4(1.14-1.73)$ & $1.27(1.04-1.55)$ & $1.26(1.03-1.54)$ & $1.21(0.99-1.49)$ \\
\hline Privacy & No & $1.58(1.24-2.02)$ & $1.24(0.98-1.57)$ & $1.55(1.22-1.97)$ & $1.34(1.05-1.7)$ \\
\hline \multicolumn{6}{|l|}{ COVID-19-related } \\
\hline $\begin{array}{l}\text { Loved one with } \\
\text { COVID }\end{array}$ & Yes & $1.41(0.99-2)$ & $1.33(0.94-1.88)$ & $1.55(1.1-2.18)$ & $1.66(1.17-2.34)$ \\
\hline $\begin{array}{l}\text { Loved one died of } \\
\text { COVID }\end{array}$ & Yes & $3.45(1.67-7.11)$ & $1.5(0.73-3.08)$ & $1.62(0.8-3.29)$ & $1.3(0.64-2.62)$ \\
\hline $\begin{array}{l}\text { Fear of getting } \\
\text { COVID }\end{array}$ & Yes & $1.11(0.92-1.33)$ & $1.46(1.22-1.73)$ & $1.13(0.95-1.35)$ & $1.5(1.25-1.8)$ \\
\hline \multirow{2}{*}{$\begin{array}{l}\text { Trust in the } \\
\text { government }\end{array}$} & Yes, enough & $0.63(0.5-0.8)$ & $0.8(0.64-1.01)$ & $0.6(0.48-0.75)$ & $0.79(0.63-1.00)$ \\
\hline & Yes, fully & $0.37(0.25-0.53)$ & $0.46(0.33-0.64)$ & $0.37(0.26-0.52)$ & $0.45(0.31-0.64)$ \\
\hline \multicolumn{6}{|l|}{ Job/economy } \\
\hline Parent's job & Still employed & $0.99(0.80-1.24)$ & $1.20(0.97-1.48)$ & $1.04(0.84-1.28)$ & $1.03(0.83-1.28)$ \\
\hline \multirow[b]{2}{*}{$\begin{array}{l}\text { Worries about } \\
\text { money }\end{array}$} & Unemployed & $0.70(0.38-1.28)$ & $1.45(0.84-2.49)$ & $1.49(0.87-2.55)$ & $1.50(0.87-2.58)$ \\
\hline & & $1.31(1.2-1.43)$ & $1.47(1.35-1.6)$ & $1.51(1.39-1.65)$ & $1.53(1.4-1.67)$ \\
\hline \multirow{3}{*}{$\begin{array}{l}\text { Relationships } \\
\text { Changed } \\
\text { relationship parents }\end{array}$} & & & & & \\
\hline & Positive & 0.87 (0.68-1.13) & 0.99 (0.78-1.27) & $0.95(0.73-1.23)$ & $1.07(0.82-1.39)$ \\
\hline & Negative & $1.09(0.77-1.53)$ & $1.55(1.1-2.2)$ & $1.1(0.77-1.56)$ & $1.19(0.84-1.7)$ \\
\hline \multirow{2}{*}{$\begin{array}{l}\text { Changed } \\
\text { relationship friends }\end{array}$} & Positive & $1.26(0.96-1.66)$ & $1.09(0.84-1.42)$ & 1.04 (0.79-1.37) & $1.14(0.86-1.51)$ \\
\hline & Negative & $1.55(1.15-2.09)$ & $1.5(1.13-2)$ & $1.4(1.04-1.88)$ & $1.7(1.26-2.3)$ \\
\hline \multirow{2}{*}{$\begin{array}{l}\text { Changed } \\
\text { relationship partner }\end{array}$} & Positive & $0.93(0.72-1.21)$ & $1.16(0.9-1.51)$ & 1.07 (0.82-1.39) & $1.17(0.89-1.52)$ \\
\hline & Negative & $0.93(0.7-1.23)$ & $1.07(0.81-1.41)$ & 1.27 (0.95-1.68) & $1.04(0.78-1.39)$ \\
\hline \multicolumn{6}{|l|}{ Social media } \\
\hline \multirow{3}{*}{$\begin{array}{c}\text { Support } \\
\text { Helpful family } \\
\text { support } \\
\text { Helpful external } \\
\text { support }\end{array}$} & Yes & $0.64(0.52-0.79)$ & $0.75(0.61-0.91)$ & $0.48(0.39-0.58)$ & $0.5(0.41-0.61)$ \\
\hline & Yes & $1.52(1.24-1.85)$ & $2.11(1.74-2.55)$ & $2.22(1.84-2.7)$ & $2.44(2.01-2.97)$ \\
\hline & Already in therapy & $1.49(0.98-2.28)$ & $1.37(0.91-2.07)$ & $2.28(1.5-3.46)$ & $2.26(1.49-3.43)$ \\
\hline \multicolumn{6}{|l|}{ Positive impact } \\
\hline New interests & Yes & $0.94(0.78-1.13)$ & $0.97(0.82-1.16)$ & $0.8(0.67-0.96)$ & $0.99(0.83-1.2)$ \\
\hline Creative time & Yes & $0.61(0.51-0.73)$ & $0.59(0.5-0.71)$ & $0.39(0.32-0.46)$ & $0.51(0.42-0.61)$ \\
\hline
\end{tabular}

Having a small house was significantly associated with anger (OR: 1.3; 95\% CI: 1.00-1.69), while not spending time outside was significantly associated with anger, sadness, and bore- 
dom/emptiness (anger: OR: 1.4; 95\% CI: 1.14-1.73; sadness OR: 1.27; 95\% CI: 1.04-1.55; and boredom/emptiness: OR: 1.26; 95\% CI: 1.03-1.54) (Table 2). Interestingly, several lockdownrelated and COVID-19-related variables were associated with emotional reactions, and thus included in the multivariable analysis (Table 2).

\subsection{Multivariable Analyses}

Table 3 reports the results of the multivariable analyses including only variables that were significantly associated with the outcomes in the univariate analysis.

Table 3. Multivariable Analysis. Logistic regression models estimating the independent association between all the variables in column 1 (associated at $p<0.05$ in the univariable analyses) and the outcomes in the last four columns; all models are also adjusted for country. Statistically significant variables are in bold.

\begin{tabular}{|c|c|c|c|c|c|}
\hline Variable & Category & $\begin{array}{c}\text { Anger } \\
\text { OR }(95 \% \mathrm{CI})\end{array}$ & $\begin{array}{c}\text { Sadness } \\
\text { OR }(95 \% \text { CI })\end{array}$ & $\begin{array}{l}\text { Boredom/Emptiness } \\
\text { OR }(95 \% \text { CI })\end{array}$ & $\begin{array}{c}\text { Anxiety } \\
\text { OR }(95 \% \mathrm{CI})\end{array}$ \\
\hline \multicolumn{6}{|l|}{ Sociodemographic } \\
\hline \multirow[t]{2}{*}{ Age } & $>16$ & $1.53(1.14-2.07)$ & $1.55(1.17-2.05)$ & $1.42(1.05-1.92)$ & $1.78(1.31-2.43)$ \\
\hline & $14-16$ & $1.59(1.19-2.11)$ & $1.67(1.28-2.18)$ & $1.41(1.06-1.87)$ & $1.59(1.18-2.13)$ \\
\hline Gender & Male & $0.76(0.61-0.94)$ & $0.4(0.33-0.5)$ & $0.71(0.57-0.89)$ & $0.55(0.44-0.69)$ \\
\hline \multirow[t]{2}{*}{ Urbanization } & $\begin{array}{l}\text { Intermediate } \\
\text { densitv areas }\end{array}$ & $0.84(0.57-1.23)$ & $1.09(0.76-1.57)$ & $1.2(0.82-1.76)$ & $0.69(0.46-1.04)$ \\
\hline & Rural & $0.72(0.43-1.22)$ & $0.74(0.45-1.21)$ & $0.95(0.56-1.61)$ & $0.41(0.21-0.78)$ \\
\hline Mother low SES & Yes & - & - & $0.80(0.65-0.98)$ & - \\
\hline \multicolumn{6}{|l|}{ Housing } \\
\hline \multirow{3}{*}{$\begin{array}{c}\text { House } \\
\text { Time outside home } \\
\text { Privacy }\end{array}$} & $<60 \mathrm{~m}^{2}$ & $1.07(0.8-1.42)$ & - & - & - \\
\hline & No & $1.33(1.06-1.66)$ & $1.3(1.04-1.63)$ & $1.17(0.93-1.48)$ & - \\
\hline & No & $1.2(0.91-1.57)$ & - & $0.98(0.74-1.3)$ & $0.94(0.71-1.24)$ \\
\hline \multicolumn{6}{|l|}{ COVID-19-related } \\
\hline $\begin{array}{l}\text { Loved one with } \\
\text { COVID }\end{array}$ & Yes & - & - & - & $1.31(0.9-1.91)$ \\
\hline $\begin{array}{l}\text { Loved one died of } \\
\text { COVID }\end{array}$ & Yes & $2.74(1.29-5.81)$ & - & $1.11(0.51-2.44)$ & - \\
\hline $\begin{array}{l}\text { Fear of getting } \\
\text { COVID }\end{array}$ & Yes & - & $1.31(1.08-1.59)$ & $1.08(0.89-1.32)$ & $1.46(1.19-1.79)$ \\
\hline \multirow{2}{*}{$\begin{array}{l}\text { Trust in the } \\
\text { government }\end{array}$} & Yes, enough & $0.72(0.57-0.93)$ & $0.87(0.67-1.12)$ & $0.71(0.55-0.93)$ & $0.95(0.73-1.23)$ \\
\hline & Yes, fully & $0.52(0.35-0.76)$ & $0.62(0.43-0.89)$ & $0.62(0.42-0.91)$ & $0.74(0.5-1.11)$ \\
\hline \multicolumn{6}{|l|}{ Job/economy } \\
\hline \multicolumn{2}{|l|}{$\begin{array}{c}\text { Worries about } \\
\text { money }\end{array}$} & $1.15(1.05-1.27)$ & $1.28(1.17-1.41)$ & $1.32(1.2-1.46)$ & $1.32(1.2-1.46)$ \\
\hline \multicolumn{6}{|l|}{ Relationships } \\
\hline \multirow{2}{*}{$\begin{array}{l}\text { Changed } \\
\text { relationship parents }\end{array}$} & Positive & $0.87(0.68-1.12)$ & $1(0.79-1.28)$ & $0.94(0.73-1.22)$ & $1.08(0.83-1.4)$ \\
\hline & Negative & $1.1(0.79-1.55)$ & $1.55(1.1-2.2)$ & $1.08(0.76-1.54)$ & $1.22(0.86-1.72)$ \\
\hline \multirow{2}{*}{$\begin{array}{l}\text { Changed } \\
\text { relationship friends }\end{array}$} & Positive & $1.27(0.97-1.67)$ & $1.08(0.83-1.4)$ & $1.04(0.79-1.37)$ & $1.14(0.86-1.5)$ \\
\hline & Negative & $1.55(1.16-2.09)$ & $1.49(1.12-1.99)$ & $1.38(1.03-1.86)$ & $1.7(1.26-2.3)$ \\
\hline \multirow{2}{*}{$\begin{array}{l}\text { Changed } \\
\text { relationship partner }\end{array}$} & Positive & $0.93(0.71-1.2)$ & $1.16(0.9-1.49)$ & $1.07(0.82-1.39)$ & $1.14(0.88-1.48)$ \\
\hline & Negative & $0.91(0.69-1.21)$ & $1.07(0.81-1.41)$ & $1.27(0.96-1.69)$ & $1.04(0.78-1.38)$ \\
\hline Increased use & & $1.18(1.09-1.29)$ \\
\hline \multirow{3}{*}{$\begin{array}{l}\text { Helpful family } \\
\text { support } \\
\text { Helpful external } \\
\text { support }\end{array}$} & Yes & $0.89(0.7-1.12)$ & $1.02(0.81-1.29)$ & $0.66(0.52-0.84)$ & $0.61(0.48-0.78)$ \\
\hline & Yes & $1.21(0.98-1.5)$ & $1.49(1.2-1.84)$ & $1.73(1.39-2.14)$ & $1.72(1.39-2.13)$ \\
\hline & Already in therapy & $1.08(0.69-1.69)$ & $0.89(0.57-1.39)$ & $1.72(1.08-2.74)$ & $1.49(0.95-2.33)$ \\
\hline \multicolumn{6}{|l|}{ Positive impact } \\
\hline New interests & Yes & - & - & $0.93(0.75-1.15)$ & - \\
\hline Creative time & Yes & $0.78(0.64-0.96)$ & $0.66(0.54-0.81)$ & $0.51(0.41-0.63)$ & $0.63(0.51-0.78)$ \\
\hline
\end{tabular}

Sociodemographic variables. Among sociodemographic variables, we found that the likelihood of reporting anger (OR: 1.59; 95\% CI: 1.19-2.11), sadness (OR: 1.67; 95\% CI: 1.28-2.18), boredom/emptiness (OR: 1.41: 95\% CI: 1.06-1.87), and anxiety (OR: 1.59: 95\% 
CI: 1.18-2.13) were higher among adolescents aged 14-16 years and among those older than 16 years, compared to those aged $<14$ years, in line with the univariable analyses. Similarly, being a boy was still independently associated with a lower likelihood of reporting all emotional reactions (Table 3). Living in a rural area was associated with a decreased likelihood of experiencing anxiety (OR: $0.41 ; 95 \%$ CI: 0.21-0.78), but the association with anger, boredom/emptiness, and sadness/depression were no longer significant in the multivariable analysis.

Housing. Adolescents reporting not spending time outside their home during the lockdown were significantly more likely to experience anger (OR: 1.33; 95\% CI: 1.06-1.66) and sadness (OR: 1.3; 95\% CI: 1.04-1.63).

COVID-19-related variables. We found that adolescents who reported that a loved person had died from COVID had a more than two times higher risk of reporting feelings of anger (OR: 2.74; 95\% CI: 1.29-5.81) compared with an adolescent who did not. Interestingly, strongly trusting the government was found to be significantly protective against experiencing anger (OR: 0.52; 95\% CI: 0.35-0.76), sadness (OR: 0.62; 95\% CI: $0.43-0.89$ ), and boredom/emptiness (OR: 0.62; 95\% CI: 0.42-0.91).

Relationships with parents and peers. We found that those reporting a negative impact of the lockdown on their relationships with friends were at higher risk of experiencing all emotional difficulties (e.g., OR for anxiety: 1.7; 95\% CI: 1.26-2.30). Additionally, adolescents reporting a negative impact on their relationships with parents were more likely to experience sadness (OR: 1.55; 95\% CI: 1.10-2.20).

Social media. We found a significantly increased likelihood of experiencing all emotional reactions among adolescents who reported increased use of social media (Table 3), especially boredom/emptiness (OR: 1.44; 95\% CI: 1.32-1.57).

Support. We found that those who believed that family support was important during the lockdown were less likely to report both boredom/emptiness (OR: 0.66; 95\% CI: 0.52-0.84) and anxiety (OR: $0.61 ; 95 \%$ CI: $0.48-0.78$ ) compared to those who did not believe so. In contrast, those who considered external support as important were more likely to report all emotional difficulties except anger. Notably, those reporting to be in psychotherapy were more likely to report boredom/emptiness (OR: 1.72; 95\% CI: 1.08-2.74).

The positive impact of the lockdown. Adolescents who reported to spend time creatively were significantly less likely to experience anger (OR: $0.78 ; 95 \%$ CI: $0.64-0.96$ ), sadness (OR: 0.66; 95\% CI: 0.54-0.81), and boredom/emptiness (OR: 0.51; 95\% CI: $0.41-0.63$ ) than those who did not spend time creatively.

\section{Discussion}

The present study reports the findings from a survey on the emotional reactions of a large sample of European secondary school students during the COVID-19 crisis. We found that the likelihood of experiencing anger, sadness, boredom/emptiness, and anxiety was higher among oldest ( $>14$ years) and female adolescents, and related to housing characteristics and time spent outside. This is partly consistent with other studies where the female gender was found to be related to higher levels of psychological distress [23-25]. Additionally, recent studies on Chinese adolescents showed that older girls (15-18 years) were more likely to present depressive/anxious symptoms [11,26]. This might be, in part, explained by the fact that girls are generally more prone to internalizing-spectrum symptoms [27], while boys might be more likely to show externalizing behaviors and underreport internalizing emotions [28]. This gender difference might inform schoolbased preventive, gender-targeted interventions; it might also suggest that the detection of internalizing emotional difficulties might be underestimated among boys, who are at higher risk of developmental difficulties and negative later mental health outcomes than girls [29], including a higher risk of suicide [30,31]. Notably, even if boys were often less likely to express emotional distress, this might be related to societal and cultural constraints resulting in underreporting of emotional difficulties [29]. This might also suggest a need for addressing healthy masculinities and gender equality in emotional expressions [32] 
We also found several protective factors. Living in a rural area was protective against experiencing anxiety, while spending time creatively during the lockdown was significantly protective of experiencing anger, sadness, and boredom/emptiness. Consistently, living in an urban area was already found to be a risk factor for experiencing anxiety among college students [33]. Thus, societal disparities, such as housing characteristics, might exacerbate the adverse emotional effects of the COVID-19 pandemic and have an impact on the emotional reactions of adolescents [34]. Our findings expand the knowledge on the protective effect of daily routine and positive reframing [35], which have been found to be protective factors against perceived stress and emotional difficulties [24]. This was also found in a previous Italian survey, which reported that reconstructing a sort of daily "agenda" during the lockdown helped the overall emotional balance of children [36].

Results from the present survey provide important insights into adolescents' emotional reaction after losing someone from COVID-19; we found that young individuals who lost a loved one from COVID-19 were at twice the risk of experiencing anger compared to those who did not (OR: 2.74; 95\% CI: 1.29-5.81). The feeling of anger was already found to be a common emotional reaction among adolescents during lockdown [24], but our study expands the knowledge by suggesting that it is important to address the feeling of anger among young people who have lost someone from COVID-19. Indeed, reacting with anger might suggest that COVID-19 related grief among adolescents is experienced with a sense of injustice, which is typical of conflict-related trauma [37]. Future research is necessary to better understand the complexity of grief reactions among adolescents who lost someone from COVID-19, as this may inform preventive and therapeutic interventions. A previous study also reported that anger was found as a traumatic reaction among the general population of the Czech Republic, and that this was related to mass media pessimism [38]. Thus, our findings also suggest that future research studies are needed to clarify the association between mass media reporting and adolescent emotional reactions.

Interestingly, we also found that increased use of social media was significantly associated with all negative emotional reactions investigated, consistently with previous studies [10,39]. However, the direction of this association is difficult to interpret, as adolescents experiencing emotional difficulties might use social media more frequently [40]. Further studies are needed, aimed at understanding the role of social media in identifying youth in need of help, who are more likely to report negative emotional reactions.

Our findings also highlight the importance of the relationship with peers; those reporting a negative impact of the lockdown on their relationship with peers were at higher risk of experiencing negative emotional reactions, particularly more anxiety. This finding is in line with previous evidence highlighting the important role of peer relationships in the development of anxiety among adolescents [41,42]. Moreover, this suggests the importance of establishing peer support networks, either facilitated by peers or by professional interventions [43-45].

Notably, our findings also showed that trusting government decision-making could be considered a protective factor against negative emotional reactions among young students; strongly trusting the government was found to be significantly protective against experiencing anger, sadness, and boredom/emptiness. Previous findings also suggested that improving knowledge and positive attitudes toward the crisis among young people might enhance their resilience and reduce the risk of the psychological burden of restrictive measures [26].

\section{Limitations}

The present findings should be interpreted in light of several limitations. First, the cross-sectional design; emotional difficulties were measured at the same assessment, during the lockdown, thus the directions of the associations described are uncertain. Moreover, students were not randomly selected, and this might limit the generalizability to the entire population. Furthermore, the overall mental health status of the respondents is not known prior to the pandemic, so self-selection to complete the survey and participate may be taken 
into account in interpreting the results. Finally, in the absence of pre-pandemic data, we cannot know if several of the reported associations (e.g., between social media and anxiety) are specifically related to the current COVID-19 crisis or are more general associations that we would have observed independently from the crisis.

\section{Conclusions}

The present survey demonstrated that the risk of experiencing anger, sadness, boredom/emptiness, and anxiety was higher among older adolescents, females, and adolescents living in a small flat, not spending time outside, and reporting increased use of social media. Losing a loved one from COVID-19 was specifically associated with anger among affected adolescents, suggesting a specific reaction to such a tragic event. Nonetheless, several protective factors were identified, such as spending time creatively during the lockdown and trusting the government's decisions. The present findings might help to identify adolescents more likely to report negative emotional reactions during the COVID-19 pandemic and inform policymakers and future public health strategies on improving mental health among adolescents. Additionally, the present study might inform future research on school-based preventive interventions, suggesting that improving trust in public health policies, social connectedness, as well as improving knowledge and positive attitudes toward the health crisis, might enhance resilience and reduce the risk of psychological burden among school students.

Author Contributions: Conceptualization, A.F., M.O., M.B. and E.M.; methodology, A.F., M.O., E.M., L.Q., S.F., O.C.; software, A.F., M.O., M.B. and P.V.; validation, A.F., M.O., M.B. and E.M.; formal analysis, A.F., M.O. and M.B.; investigation, all authors; resources, all authors; data curation, all authors; writing-Original draft preparation, A.F., M.O., M.B., P.V., C.I. and E.M.; writing-Review and editing, A.F., M.O., M.B. and E.M.; visualization, A.F., M.O., M.B., P.V., D.L. and E.M.; supervision, M.O.; and project administration, all authors. All authors have read and agreed to the published version of the manuscript.

Funding: This research received no external funding.

Institutional Review Board Statement: The project received Institutional approval by the Municipality of Rome, Department of School and Education (institutional authorization number n.987 06/05/2019), and was considered in line with GDPR 2016/679 (General Data Protection Regulation).

Informed Consent Statement: Informed consent was obtained from all subjects involved in the study.

Data Availability Statement: The data that support the findings of this study are available on request from the corresponding author. The data are not publicly available due to privacy or ethical restrictions.

Acknowledgments: The authors would like to thank Giuseppina Pica and the Department for School Politics of the City of Rome, Marco Gusman, Alessia Barbagli, Valentina Bianchini, Marijana Gasparovic, Mirela Groza, Hanny Karlic, Miljana Kukic, Christina Kulterer, Mirela Lăpugean, Caterina Medici M.D., Francesca Padrevecchi, Fabiana Piccinini, Cecilia Santi, Sandra Santomauro, Carla Severini, Laura Soave, Andrew Stephens, Ramona Daniela Varga, Melanie Vicevic, and Lorenzo Webster for their helpful contribution in translating and distributing the questionnaire. A special thanks to headteachers, teachers, and students who took part in the present survey.

Conflicts of Interest: The authors declare no conflict of interest.

\section{References}

1. WHO. Coronavirus Disease 2019 (COVID-19) Situation Report-70; WHO: Geneva, Switzerland, 2020; Volume 14.

2. Fegert, J.M.; Vitiello, B.; Plener, P.L.; Clemens, V. Challenges and burden of the Coronavirus 2019 (COVID-19) pandemic for child and adolescent mental health: A narrative review to highlight clinical and research needs in the acute phase and the long return to normality. Child Adolesc. Psychiatry Ment. Health 2020, 14, 20. [CrossRef] [PubMed]

3. UNESCU School Closures Caused by Coronavirus (COVID-19). Available online: https://en.unesco.org/covid19/ educationresponse (accessed on 1 September 2020).

4. Zhang, L.; Zhang, D.; Fang, J.; Wan, Y.; Tao, F.; Sun, Y. Assessment of Mental Health of Chinese Primary School Students before and after School Closing and Opening during the COVID-19 Pandemic. JAMA Netw. Open 2020, 3, e2021482. [CrossRef] [PubMed] 
5. Hoekstra, P.J. Suicidality in children and adolescents: Lessons to be learned from the COVID-19 crisis. Eur. Child Adolesc. Psychiatry 2020, 29, 737-738. [CrossRef] [PubMed]

6. Loades, M.E.; Chatburn, E.; Higson-Sweeney, N.; Reynolds, S.; Shafran, R.; Brigden, A.; Linney, C.; McManus, M.N.; Borwick, C.; Crawley, E. Rapid Systematic Review: The Impact of Social Isolation and Loneliness on the Mental Health of Children and Adolescents in the Context of COVID-19. J. Am. Acad. Child Adolesc. Psychiatry 2020, 59, 1218-1239. [CrossRef]

7. Ali, M.M.; West, K.; Teich, J.L.; Lynch, S.; Mutter, R.; Dubenitz, J. Utilization of Mental Health Services in Educational Setting by Adolescents in the United States. J. Sch. Health 2019, 89, 393-401. [CrossRef]

8. Golberstein, E.; Gonzales, G.; Meara, E. How do economic downturns affect the mental health of children? Evidence from the National Health Interview Survey. Health Econ. 2019, 28, 955-970. [CrossRef]

9. Magson, N.R.; Freeman, J.Y.A.; Rapee, R.M.; Richardson, C.E.; Oar, E.L.; Fardouly, J. Risk and Protective Factors for Prospective Changes in Adolescent Mental Health during the COVID-19 Pandemic. J. Youth Adolesc. 2020, 50, 44-57. [CrossRef]

10. Guessoum, S.B.; Lachal, J.; Radjack, R.; Carretier, E.; Minassian, S.; Benoit, L.; Moro, M.R. Adolescent psychiatric disorders during the COVID-19 pandemic and lockdown. Psychiatry Res. 2020, 291, 113264. [CrossRef] [PubMed]

11. Zhou, S.J.; Zhang, L.G.; Wang, L.L.; Guo, Z.C.; Wang, J.Q.; Chen, J.C.; Liu, M.; Chen, X.; Chen, J.X. Prevalence and sociodemographic correlates of psychological health problems in Chinese adolescents during the outbreak of COVID-19. Eur. Child Adolesc. Psychiatry 2020, 29, 749-758. [CrossRef]

12. Chi, X.; Becker, B.; Yu, Q.; Willeit, P.; Jiao, C.; Huang, L.; Hossain, M.M.; Grabovac, I.; Yeung, A.; Lin, J.; et al. Prevalence and Psychosocial Correlates of Mental Health Outcomes among Chinese College Students during the Coronavirus Disease (COVID-19) Pandemic. Front. Psychiatry 2020, 11, 1. [CrossRef]

13. Taquet, M.; Quoidbach, J.; Fried, E.I.; Goodwin, G.M. Mood Homeostasis before and during the Coronavirus Disease 2019 (COVID-19) Lockdown among Students in the Netherlands. JAMA Psychiatry 2020, 78, 110-112. [CrossRef]

14. Akkaya-Kalayci, T.; Kothgassner, O.D.; Wenzel, T.; Goreis, A.; Chen, A.; Ceri, V.; Özlü-Erkilic, Z. The Impact of the COVID-19 Pandemic on Mental Health and Psychological Well-Being of Young People Living in Austria and Turkey: A Multicenter Study. Int. J. Environ. Res. Public Health 2020, 17, 9111. [CrossRef] [PubMed]

15. De Oliveira, W.A.; da Silva, J.L.; Andrade, A.L.M.; de Micheli, D.; Carlos, D.M.; Silva, M.A.I. Adolescents' health in times of COVID-19: A scoping review. Cad. Saude Publica 2020, 36. [CrossRef] [PubMed]

16. Hawes, M.T.; Szenczy, A.K.; Klein, D.N.; Hajcak, G.; Nelson, B.D. Increases in Depression and Anxiety Symptoms in Adolescents and Young Adults during the COVID-19 Pandemic. Psychol. Med. 2021. [CrossRef] [PubMed]

17. Zhang, C.; Ye, M.; Fu, Y.; Yang, M.; Luo, F.; Yuan, J.; Tao, Q. The Psychological Impact of the COVID-19 Pandemic on Teenagers in China. J. Adolesc. Health 2020, 67, 747-755. [CrossRef]

18. Qin, Z.; Shi, L.; Xue, Y.; Lin, H.; Zhang, J.; Liang, P.; Lu, Z.; Wu, M.; Chen, Y.; Zheng, X.; et al. Prevalence and Risk Factors Associated with Self-reported Psychological Distress Among Children and Adolescents during the COVID-19 Pandemic in China. JAMA Netw. Open 2021, 4, e2035487. [CrossRef]

19. Guerrero-Gomez, A.; Nöthen-Garunja, I.; Schredl, M.; Homberg, A.; Vulcan, M.; Brusić, A.; Bonizzi, C.; Iannaco, C. Dreaming in Adolescents during the COVID-19 Health Crisis: Survey Among a Sample of European School Students. Front. Psychol. 2021, 12, 1054. [CrossRef]

20. Homberg, A. Schedati, perseguitati, sterminati-Die Ausstellung in Italien. In Erfasst, Verfolgt, Vernichtet. Kranke und Behinderte im Nationalsozialismus; Schneider, F., Ed.; Springer: Berlin/Heidelberg, Germany, 2019; pp. 113-129.

21. Degree of Urbanisation Classification-2011 Revision-Statistics Explained. Available online: https: / ec.europa.eu/eurostat/ statistics-explained/index.php/Degree_of_urbanisation_classification_-_2011_revision (accessed on 11 June 2020).

22. Lien, N.; Friestad, C.; Klepp, K.I. Adolescents' proxy reports of parents' socioeconomic status: How valid are they? J. Epidemiol. Community Health 2001, 55, 731-737. [CrossRef] [PubMed]

23. Horesh, D.; Kapel Lev-Ari, R.; Hasson-Ohayon, I. Risk factors for psychological distress during the COVID-19 pandemic in Israel: Loneliness, age, gender, and health status play an important role. Br. J. Health Psychol. 2020, 25, 925-933. [CrossRef]

24. Shanahan, L.; Steinhoff, A.; Bechtiger, L.; Murray, A.L.; Nivette, A.; Hepp, U.; Ribeaud, D.; Eisner, M. Emotional Distress in Young Adults during the COVID-19 Pandemic: Evidence of Risk and Resilience from a Longitudinal Cohort Study. Psychol. Med. 2020. [CrossRef] [PubMed]

25. Terry, P.C.; Parsons-Smith, R.L.; Terry, V.R. Mood Responses Associated With COVID-19 Restrictions. Front. Psychol. 2020, 11, 3090. [CrossRef] [PubMed]

26. Zhou, J.; Yuan, X.; Qi, H.; Liu, R.; Li, Y.; Huang, H.; Chen, X.; Wang, G. Prevalence of depression and its correlative factors among female adolescents in China during the coronavirus disease 2019 outbreak. Glob. Health 2020, 16, 69. [CrossRef] [PubMed]

27. Duffy, M.E.; Twenge, J.M.; Joiner, T.E. Trends in Mood and Anxiety Symptoms and Suicide-Related Outcomes among U.S. Undergraduates, 2007-2018: Evidence from Two National Surveys. J. Adolesc. Health 2019, 65, 590-598. [CrossRef]

28. Chaplin, T.M.; Aldao, A. Gender differences in emotion expression in children: A meta-analytic review. Psychol. Bull. 2013, 139, 735-765. [CrossRef] [PubMed]

29. Kraemer, S. The fragile male. Br. Med. J. 2000, 321, 1609-1612. [CrossRef] [PubMed]

30. Ruch, D.A.; Sheftall, A.H.; Schlagbaum, P.; Rausch, J.; Campo, J.V.; Bridge, J.A. Trends in Suicide among Youth Aged 10 to 19 Years in the United States, 1975 to 2016. JAMA Netw. Open 2019, 2, e193886. [CrossRef] [PubMed] 
31. Pompili, M.; Vichi, M.; De Leo, D.; Pfeffer, C.; Girardi, P. A longitudinal epidemiological comparison of suicide and other causes of death in Italian children and adolescents. Eur. Child Adolesc. Psychiatry 2012, 21, 111-121. [CrossRef] [PubMed]

32. Randell, E.; Jerdén, L.; Öhman, A.; Starrin, B.; Flacking, R. Tough, sensitive and sincere: How adolescent boys manage masculinities and emotions. Int. J. Adolesc. Youth 2016, 21, 486-498. [CrossRef]

33. Cao, W.; Fang, Z.; Hou, G.; Han, M.; Xu, X.; Dong, J.; Zheng, J. The psychological impact of the COVID-19 epidemic on college students in China. Psychiatry Res. 2020, 287, 112934. [CrossRef] [PubMed]

34. Horton, R. Offline: COVID-19 is not a pandemic. Lancet 2020, 396, 874. [CrossRef]

35. Ren, H.; He, X.; Bian, X.; Shang, X.; Liu, J. The Protective Roles of Exercise and Maintenance of Daily Living Routines for Chinese Adolescents during the COVID-19 Quarantine Period. J. Adolesc. Health 2020, 68, 35-42. [CrossRef]

36. Buzzi, C.; Tucci, M.; Ciprandi, R.; Brambilla, I.; Caimmi, S.; Ciprandi, G.; Marseglia, G.L. The psycho-social effects of COVID-19 on Italian adolescents' attitudes and behaviors. Ital. J. Pediatr. 2020, 46, 4-10. [CrossRef] [PubMed]

37. Tay, A.K.; Rees, S.; Steel, Z.; Liddell, B.; Nickerson, A.; Tam, N.; Silove, D. The role of grief symptoms and a sense of injustice in the pathways to post-traumatic stress symptoms in post-conflict Timor-Leste. Epidemiol. Psychiatr. Sci. 2017, 26, 403-413. [CrossRef] [PubMed]

38. Trnka, R.; Lorencova, R. Fear, Anger, and Media-Induced Trauma during the Outbreak of COVID-19 in the Czech Republic. Psychol. Trauma Theory Res. Pract. Policy 2020, 12, 546. [CrossRef]

39. Gao, J.; Zheng, P.; Jia, Y.; Chen, H.; Mao, Y.; Chen, S.; Wang, Y.; Fu, H.; Dai, J. Mental health problems and social media exposure during COVID-19 outbreak. PLoS ONE 2020, 15, e0231924.

40. Fernandes, B.; Biswas, U.N.; Tan-mansukhani, R.; Vallejo, A.; Essau, C.A. The impact of COVID-19 lockdown on internet use and escapism in adolescents. Rev. Psicol. Clínica Niños Adolesc. 2020, 7, 59-65.

41. La Greca, A.M.; Harrison, H.M. Adolescent peer relations, friendships, and romantic relationships: Do they predict social anxiety and depression? J. Clin. Child Adolesc. Psychol. 2005, 34, 49-61. [CrossRef] [PubMed]

42. Pickering, L.; Hadwin, J.A.; Kovshoff, H. The Role of Peers in the Development of Social Anxiety in Adolescent Girls: A Systematic Review. Adolesc. Res. Rev. 2020, 5, 341-362. [CrossRef]

43. Thakur, A. Mental Health in High School Students at the Time of COVID-19: A Student's Perspective. J. Am. Acad. Child Adolesc. Psychiatry 2020, 59, 1309-1310. [CrossRef]

44. Scardera, S.; Perret, L.C.; Ouellet-Morin, I.; Gariépy, G.; Juster, R.-P.; Boivin, M.; Turecki, G.; Tremblay, R.E.; Côté, S.; Geoffroy, M.-C. Association of Social Support during Adolescence with Depression, Anxiety, and Suicidal Ideation in Young Adults. JAMA Netw. Open 2020, 3, e2027491. [CrossRef] [PubMed]

45. Qi, M.; Zhou, S.J.; Guo, Z.C.; Zhang, L.G.; Min, H.J.; Li, X.M.; Chen, J.X. The Effect of Social Support on Mental Health in Chinese Adolescents during the Outbreak of COVID-19. J. Adolesc. Health 2020, 67, 514-518. [CrossRef] [PubMed] 